\title{
Vitamin D Supplementation for Depressive Symptoms: A Systematic Review and Meta-analysis of Randomized Controlled Trials
}

\author{
Jonathan A. Shaffer, PhD, MS Biostats ${ }^{1}$, Donald Edmondson, PhD, MPH ${ }^{1}$, Lauren Taggart \\ Wasson, MD, MPH ${ }^{1}$, Louise Falzon, PGDiplnf ${ }^{1}$, Kirsten Homma, BA ${ }^{1}$, Nchedcochukwu \\ Ezeokoli ${ }^{2}$, Peter Li, BA ${ }^{3}$, and Karina W. Davidson, PhD ${ }^{1}$ \\ ${ }^{1}$ Center for Behavioral Cardiovascular Health, Columbia University Medical Center, NY, NY \\ 2Department of Chemistry, School of Humanities \& Sciences, Stanford University, Stanford, CA \\ ${ }^{3}$ Department of Mathematics, College of Arts and Sciences, New York University, NY, NY
}

\begin{abstract}
Objective-To review the effects of vitamin D supplementation on depression or depressive symptoms in randomized controlled trials. Although low vitamin D levels have been observationally associated with depression and depressive symptoms, the effect of vitamin D supplementation as an antidepressant remains uncertain.
\end{abstract}

METHODS-MEDLINE, CINAHL, Allied and Complimentary Medicine Database, PsycINFO, Scopus, and The Cochrane Library, and references of included reports (through May 2013) were searched. Two independent reviewers identified randomized trials that compared the effect of vitamin D supplementation on depression or depressive symptoms to a control condition. Two additional reviewers independently reviewed and extracted relevant data; disagreements were reconciled by consensus. The Cochrane Risk of Bias Tool was used to assess study quality. Seven trials (3191 participants) were included.

RESULTS-Vitamin D supplementation had no overall effect on depressive symptoms (standardized mean difference [SMD], $-0.14 ; 95 \% \mathrm{CI},-0.33$ to $0.05 ; P=0.16$ ), although considerable heterogeneity was observed. Subgroup analysis showed that vitamin D supplementation for participants with clinically significant depressive symptoms or depressive disorder had a moderate, statistically significant effect (2 studies: SMD, $-0.60 ; 95 \%$ CI, -1.19 to $-0.01 ; \mathrm{P}=0.046)$, but a small, nonsignificant effect for those without clinically significant depression (5 studies: SMD, $-0.04 ; \mathrm{CI},-0.20$ to $0.12 ; \mathrm{P}=0.61$ ). Most trials had unclear or high risk of bias. Studies varied in the amount, frequency, duration, and mode of delivery of vitamin D supplementation.

Conclusion-Vitamin D supplementation may be effective for reducing depressive symptoms in patients with clinically significant depression; however, further high quality research is needed.

\footnotetext{
Address for Correspondence: Jonathan A. Shaffer, PhD, MSBiostats Columbia University Medical Center, Center for Behavioral Cardiovascular Health 622 West 168th Street, PH9-318 New York, NY 10032 js3742@ columbia.edu; Phone: 212-342-4492; Fax: 212-305-3172.

Conflicts of Interest: The authors have no conflicts of interest to report.
} 


\section{Keywords}

vitamin D; depression; depressive symptoms; randomized controlled trials; meta-analysis; systematic review

Depression is a highly prevalent and debilitating chronic illness that can be difficult to treat $(1,2)$, and both depressive disorders and subthreshold depressive symptoms are associated with significant disability, mortality, and health care costs (3, 4). Although the underlying pathophysiology of depression remains unknown and probably involves several mechanisms, a possible role of vitamin $\mathrm{D}$ in depression has received considerable attention (5). Indeed, a recent systematic review and meta-analysis (6) of case-control, crosssectional, and prospective observational cohort studies of depression and vitamin D provided some support for an association of depression with low concentrations of serum 25-hydroxyvitamin $\mathrm{D}(25[\mathrm{OH}] \mathrm{D})$, the primary circulating form of vitamin $\mathrm{D}$ that is used to determine a patient's vitamin D status (7). Although these findings are compelling, the most important questions concerning the association of vitamin D with depression are (1) is the association causal, and (2) does vitamin D supplementation affect depressive symptom level?

We conducted a systematic review and meta-analysis of randomized controlled trials to investigate whether vitamin D supplementation improves -- or potentially worsens-depressive disorder or depressive symptoms. On the basis of previous narrative reviews (8, 9), we hypothesized that vitamin D supplementation would have a minimal effect on depression in these trials.

\section{Methods}

We followed the Cochrane Handbook for Systematic Reviews to plan and conduct this metaanalysis (10), and we report our findings according to the Preferred Reporting Items for Systematic Reviews and Meta-Analyses (PRISMA) guidelines (11).

\section{Data Sources and Searches}

We systematically identified all randomized controlled trials that examined the effect of vitamin D supplementation on depressive disorder or depressive symptoms. Although it is difficult to detect treatment effects in those with few, if any, baseline depressive symptoms (12), we nonetheless included studies of both nondepressed and depressed individuals because of our interest in determining whether vitamin D supplementation either worsened or improved depression. Potentially relevant articles were identified by searching the biomedical electronic databases Ovid MEDLINE, The Cochrane Library, Cumulative Index to Nursing and Allied Health Literature, Allied and Complimentary Medicine Database, PsycINFO, and Scopus. Dates were searched from inception to the second week of May 2013. Registers of clinical trials were searched for unpublished and ongoing studies. The initial search was conducted on June 1,2012, and weekly searches were conducted thereafter through May 15, 2013. All relevant subject headings and free-text terms were used to represent vitamin $\mathrm{D}$ and depression. Additional records were identified by searching 
the reference lists of relevant studies and reviews and by using the Related Articles feature in PubMed and the Cited Reference Search in ISI Web of Science. The search did not have any language or year restrictions, and we considered all studies regardless of their publication status. The exact search terms and search strategies for each database are reported in Table S1 in Supplemental Digital Content 1.

To determine the studies to be included in the meta-analysis, 2 trained reviewers (N.E., P.L.) independently read the title and/or abstract of every record retrieved. All potentially relevant articles were investigated as full text, and differences in opinion between the 2 reviewers were resolved by consensus or in consultation with one of the authors (J.A.S.).

\section{Database Extraction and Quality Assessment}

Two additional reviewers (L.F., K.H.) worked independently of each other and in consultation with the first author to extract relevant data from each report. These data included study characteristics (setting, design, randomization, masking, intent-to-treat analyses, sample size, trial entry criteria related to depression and vitamin $\mathrm{D}$, and primary depression measure), participant demographic characteristics (age and sex), and clinical characteristics (baseline concentration of $25[\mathrm{OH}] \mathrm{D}$ and depression status). Additional data were extracted to characterize the type, amount, frequency, duration, and mode of delivery of vitamin D supplementation, type of control conditions, and trial requirements regarding the use of nonstudy vitamin D supplementation. Study quality was assessed using the Cochrane Risk of Bias Tool (13), which considers the reporting and adequacy of random sequence generation, randomization concealment, masking of participants, research personnel, and outcome assessors, and methods for dealing with participants who were lost to follow-up or had missing data for other reasons.

Data on mean (SD) depressive symptoms were extracted as the primary end point given that no studies included a diagnosis of depressive disorder as an end point. We used available data to calculate change-from-baseline differences within and between treatments. Change scores were standardized using the SD of change. Two studies $(14,15)$ reported results as mean (SD) preintervention and postintervention depressive symptom scores but did not provide estimates of the pre-post correlation of depression scores that are required to compute effect sizes. We attempted to contact study authors to request these additional data but ultimately estimated the pre-post correlation of depression scores using published data $(16,17)$. Two studies included 2 intervention groups with different doses of vitamin D supplementation $(14,18)$, and 1 study included 2 control groups $(15)$. We pooled means and SDs across the 2 intervention and control groups in these studies to calculate effect sizes.

\section{Statistical Analysis}

Data were entered into an electronic database and analyzed using Comprehensive Meta Analysis (version 2.0; BioStat Software, Englewood, NJ) (19). We weighted each study's effect size using the inverse variance method. To summarize intervention effects across trials, we pooled data in random-effects models, which provide more conservative summary effect estimates than fixed-effects models even in the absence of statistically significant between-study heterogeneity (20). Data are expressed as standardized mean differences 
(SMDs) and 95\% CIs for the primary end point of depressive symptoms. The magnitude of intervention effects was characterized as small ( $\mathrm{SMD}=0.2)$, medium $(\mathrm{SMD}=0.5)$, and large ( $\mathrm{SMD}=0.8$ ) according to Cohen's recommendations (21).

Between-study heterogeneity was assessed using the Cochrane Q statistic, with a significance level set at $P<.10$. The magnitude of heterogeneity was evaluated using the $I^{2}$ statistic, and values of $50 \%$ or greater were considered indicative of substantial heterogeneity (10). Post hoc subgroup analyses were conducted for baseline depression status (trials of participants with clinically significant depressive symptoms or major depressive disorder vs trials that excluded such participants or whose participants had baseline depressive symptom scores indicative of no or mild depression $[22,23]$ ) and baseline vitamin D status (insufficient vs sufficient [7]). Mixed-effects analyses, in which random-effects models are used to combine studies within subgroups, were used to conduct subgroup analyses, and the $\mathrm{Q}$ statistic was calculated to compare intervention effects among studies.

We conducted sensitivity analyses in which we substituted a range of pre-post correlations ( $r$ $=0.1,0.4,0.8$, and 0.9 ) in depressive symptoms for the 2 studies that did not report these data. An additional sensitivity analysis was conducted that excluded an unpublished thesis that qualified for inclusion in our meta-analysis (15).

Although the validity of procedures for detecting publication bias is limited when the number of studies is as small as in the current meta-analysis (24), we planned to inspect funnel plots and compute Rosenthal's fail-safe $N$, which provides an estimate of the number of missing studies with nonsignificant effects that would be needed to make a significant $P$ value for the observed aggregate effect nonsignificant (25). Given that we obtain a nonsignificant overall effect, however, we did not conduct these assessments.

\section{RESULTS}

\section{Search Results}

The search for randomized controlled trials of vitamin D supplementation for depressive disorder or depressive symptoms identified 2394 reports. Details of the study flow are documented in Figure S1, Supplemental Digital Content 1. Of the 1829 nonduplicate articles identified by the initial search, 1797 were deemed ineligible or irrelevant on the basis of their titles and abstracts; the remaining 32 articles, in addition to 2 articles $(18,26)$ that were identified after the completion of the initial search through weekly database searches, required full reading. Of these 34 potentially eligible articles, 7 randomized controlled trials $(14,15,16,26-29)$ met our criteria for inclusion. Nearly all studies that were excluded at the full-text stage of review did not feature intervention designs; however, we excluded 3 intervention studies that did not feature randomization (30), did not include a depression outcome measure (31), or for which no published data could be identified (32).

\section{Trial Characteristics}

Table 1 and Table 2 detail the characteristics of the 7 randomized controlled trials identified by our search that examined the effect of vitamin $\mathrm{D}$ supplementation for depressive 
symptoms (total $\mathrm{N}=3191$; age range, $18-79$ years) by participant and study characteristics and depression and vitamin D trial entry criteria, respectively. All trials were published between 2003 and 2013. Two studies required that participants have low levels of 25(OH)D at baseline $(18,29)$, and participants in a third study $(15)$ of older adults also had baseline concentrations of 25(OH)D consistent with definitions of vitamin D deficiency $(<50$ $\mathrm{nmol} / \mathrm{L}$ ). Five trials either did not specifically recruit participants with depression (15) or excluded those with depressive disorders, elevated depressive symptoms, and/or current antidepressant use $(14,27$ - 29). The baseline depressive symptom scores of the participants in these 5 trials suggest that they had no depressive disorder or minimal, nonclinically significant depressive symptoms $(22,23)$. The primary end point for all 7 studies was depressive symptom scores, although the specific instruments used to assess depressive symptoms varied.

Characteristics of the vitamin D supplementation used in each of the 7 randomized controlled trials included in this review are reported in Table 3. All but one study (18) specified vitamin $\mathrm{D}_{3}$ (cholecalciferol) as the type of supplement. Mode of delivery, dosage (range, 600-300,000 IU), frequency (daily vs weekly vs one-time administration), and duration (range, 6 weeks to 2 years) of supplementation varied between studies, as did types of control conditions and requirements regarding the use of nonstudy vitamin $\mathrm{D}$ supplementation.

Assessment of study quality with the Cochrane Risk of Bias Tool demonstrated at least one unclear or high risk of bias in all but 2 trials (Table S2, Supplemental Digital Content 1) (28, 29). The most common types of bias pertained to randomization concealment $(14,15,16$, $26,27)$ and masking of research personnel $(14,15,18,26,27)$, which were rated as posing a high or unclear risk in 5 of 7 trials.

\section{Effect of Vitamin D Supplementation for Depressive Symptoms}

The overall reduction in depressive symptoms associated with vitamin D supplementation was small and nonsignificant (SMD, $-0.14 ; 95 \% \mathrm{CI},-0.33$ to $0.05 ; P=0.16$ ) (Figure). Analyses of heterogeneity revealed substantial variation among intervention effects $\left(\mathrm{Q}_{6}=\right.$ 20.2, $\left.P=0.003, I^{2}=70.3\right)$, and SMDs ranged from $-0.96(P=0.004)$ in favor of vitamin D supplementation to $0.15(P=0.49)$ in favor of control.

Subgroup analyses were conducted to identify potential sources of heterogeneity among intervention effects (Figure). The 4 studies of participants whose baseline vitamin D status was sufficient ( $>50 \mathrm{nmol} / \mathrm{L}$ ) showed a larger reduction in depressive symptoms (SMD, -0.22 ; $95 \% \mathrm{CI},-0.53$ to $0.08 ; P=0.15$ ) than the 3 studies of participants whose baseline vitamin D status was insufficient (SMD, $-0.05 ; 95 \% \mathrm{CI},-0.31$ to $0.20 ; P=0.69$ ); however, the difference in intervention effects between these 2 subgroups of studies was not significant $\left(\mathrm{Q}_{1}=0.70, P=0.40\right)$ and neither subgroup of studies had a statistically significant intervention effect.

A post hoc subgroup analysis was also conducted to compare studies of participants with clinically significant depressive symptoms and/or major depressive disorder with those that either explicitly excluded participants with clinically significant depression or included 
participants with nonclinically significant depressive symptoms at baseline (Figure). These analyses revealed that the effect of vitamin D supplementation on depressive symptoms was moderate and statistically significant in the 2 studies of participants with clinically significant depressive symptoms and/or major depressive disorder (SMD, $-0.60 ; 95 \% \mathrm{CI}$, -1.19 to $-0.01 ; P=0.046)$. In contrast, the effect of vitamin D supplementation on depressive symptoms among trials of nonclinically depressed participants was small and not statistically significant (SMD, $-0.04 ; 95 \% \mathrm{CI},-0.20$ to $0.12 ; P=0.61$ ). The difference in intervention effects between these 2 subgroups approached statistical significance $\left(\mathrm{Q}_{1}=\right.$ $3.22, P=0.07)$. We planned to investigate further sources of heterogeneity by conducting subgroup analyses of dose; however, the use of different amounts, frequencies, and durations of vitamin $\mathrm{D}$ in each trial precluded this analysis.

Sensitivity analyses, in which a range of pre-post correlations among depressive symptom scores were substituted for the published estimates used in the primary analyses, did not change the statistical significance of the overall intervention effect or the analyses of between-study heterogeneity among effects. Removal of the unpublished thesis from our analyses also did not change the primary results.

\section{Discussion}

This systematic review and meta-analysis report is the first to examine the effect of vitamin D supplementation on depressive symptoms. We found that vitamin D supplementation neither worsened nor improved depressive symptoms across 7 randomized controlled trials, but considerable heterogeneity of study characteristics and intervention effects among studies was observed. Although baseline vitamin D status did not explain the between-study heterogeneity in intervention effects, baseline depression status may have. Whereas vitamin D supplementation was associated with a statistically significant, moderate reduction in depressive symptoms across 2 trials that recruited patients with clinically significant depressive symptoms and/or major depressive disorder, its effect in trials of participants with nonclinically significant depression was small and nonsignificant.

Notwithstanding the biological plausibility of a causal role for vitamin D deficiency in depression (33), the results of this review suggest that the use of vitamin D supplementation to reduce depressive symptoms for individuals without clinicallysignificant depression may not be warranted. Although trials of nonclinically depressed individuals differed considerably in the type of participants they included, their study locations and designs, and characteristics of their intervention and control conditions, 4 of these 5 trials had nonsignificant intervention effects $(15,27-29)$. These null findings are not entirely surprising given that the association of vitamin $\mathrm{D}$ with depressive symptoms has not clearly been established in nondepressed individuals. Although a recently conducted meta-analysis of observational studies of vitamin D deficiency and depression in older adults found a moderate and statistically significant association of lower vitamin D levels with clinically meaningful depression in cross-sectional studies, the studies included in that review had several methodologic biases (6). In particular, cross-sectional studies cannot rule out the possibility of reverse causation in which patients with subthreshold depressive symptoms or depressive disorders have less exposure to sunlight and thus lower vitamin D levels (9). 
Interestingly, the same meta-analysis included 3 prospective cohort studies (34-36) that found a statistically significant, 2 -fold increased risk of developing clinically significant depression or depressive symptoms among those with low vitamin $\mathrm{D}$ levels. To date, however, no study has examined whether vitamin D supplementation offsets the risk of incident depressive disorder or depressive episodes, and future randomized controlled trials may thus be needed to do so.

Of note, not all trials of nonclinically depressed participants in this review featured null intervention effects. A trial conducted by Jorde and colleagues (14), which included participants with overweight and obesity, found a small but statistically significant reduction in depressive symptoms with vitamin D supplementation. This trial had an unclear risk of bias in 3 of the 6 domains of the Cochrane Risk of Bias Tool; however, its findings suggest a possible need for additional studies that examine mechanistic aspects of the association of vitamin $\mathrm{D}$ with depressive symptoms and vitamin $\mathrm{D}$ intervention effects in this distinct population. These findings also hint that overweight and obesity may contribute to some of the observed heterogeneity of effects among the studies included in this meta-analysis, although we could not test this hypothesis given a lack of reported data on overweight and obesity across trials.

Although our subgroup analysis of trials with vs without participants with clinically significant depressive symptoms and/or major depressive disorder suggests a possible explanation for the heterogeneity of intervention effects observed in overall analyses, several characteristics other than participants' baseline depression status differed between the former trials and the latter ones. In particular, characteristics of the vitamin D interventions used in all 7 trials varied, and no 2 studies featured the same dose or duration of vitamin D supplementation. In addition, the trial in which we observed the largest effect of vitamin D supplementation on depressive symptoms not only included participants with major depressive disorder and elevated depressive symptoms (26) but also used vitamin D supplementation as an adjunctive intervention to pharmacotherapy with fluoxetine. The other trial of participants with clinically significant depression used a dose of vitamin D that far exceeds the single, but not necessarily cumulative, doses featured in other studies (18). Vitamin D supplementation was also administered via intramuscular injection in that trial, whereas other trials included in this review administered supplementation via capsule or food. The interaction between vitamin D supplementation and selective serotonin reuptake inhibitors such as fluoxetine, the comparative efficacy of different vitamin D dose amounts, and the implications of using alternate modes of administration of vitamin D supplementation thus remain unknown, and require investigation in future trials.

As a parallel to the present study, it is worth examining studies evaluating the efficacy of omega-3 supplements for depression, which resemble studies of vitamin $\mathrm{D}$ for depression in several ways. As with studies of vitamin $\mathrm{D}$ for depression, a large proportion of omega-3 trials involve healthy participants or those with subclinical depression (37). Meta-analyses of omega-3 for depression have pooled across these studies and those of participants with clinical depression (37) and concluded that the efficacy of omega-3 for depression is stronger in clinical samples than in nonclinical ones. Similar to the results of one of the studies (26) included in this review, the effect of omega-3 on depression may also be 
stronger when used to supplement traditional antidepressants rather than as monotherapy (38). Most importantly, meta-analyses of studies of omega-3 for depression have helped to guide subsequent research, as we hope the current meta-analysis will likewise do.

Several limitations of the current review warrant attention. First, we identified few trials overall, the design characteristics of each of these studies differed considerably, and all but 2 of these trials $(28,29)$ had at least one unclear or high risk of bias. Although the heterogeneity among studies is indeed striking, it is not unlike the heterogeneity observed among studies of vitamin D for other conditions (39). The overall quality of the evidence from each trial is thus low and poses uncertainty regarding the true effect of vitamin D supplementation on depressive symptoms. Although it is unlikely that poor methodologic quality biased the results of trials of nondepressed participants toward the null hypothesis of no intervention effect, it may have inflated the treatment effects observed in the 2 trials of participants with clinically significant depression. Second, some of the decisions that we made while conducting our review may limit the validity of our findings. Although we drafted a protocol and planned extensively before conducting our review and analyses, we did not register the protocol or anticipate in advance all of the analyses that we conducted. In particular, we performed 2 post hoc subgroup analyses given that we could not conduct an a priori analysis of whether differences in vitamin $\mathrm{D}$ dose contributed to potential heterogeneity among intervention effects. Nonetheless, these post hoc analyses were informed by reasoned clinical and empirical considerations, and we did not conduct an excessive number of these analyses. A third limitation is that we did not consider whether vitamin D supplementation increased levels of $25(\mathrm{OH}) \mathrm{D}$ in each trial, and it is possible that the null effects seen in some trials reflect a failure of the intervention to improve vitamin D status.

The small number of studies included in this review, the considerable heterogeneity among these studies, and the unlikely possibly of detecting intervention effects among nonclinical samples (12) may lead one to wonder whether a systematic review and meta-analysis of vitamin D supplementation for depression at this time is premature. Given the recently published meta-analysis of observational studies of vitamin D deficiency and depression (6), we believe that now is precisely the time to highlight the dearth of evidence for a causal role of vitamin D in relation to depression, and point to the necessary next steps to determine whether any clinical benefit is likely to be gained by vitamin D supplementation.

Notwithstanding these limitations and considerations, this systematic review and metaanalysis report represents a timely contribution to the emerging literature on vitamin D and depression that may inform the development of future clinical trials. Although we found a nonsignificant effect on depressive symptoms associated with vitamin D supplementation, the intervention effects across the 7 randomized controlled trials included in this review varied significantly and considerably. We observed suggestive evidence that vitamin D supplementation may be effective for participants with major depressive disorder or subthreshold, clinically significant depressive symptoms but not for those without; however, other potential sources of the between-study heterogeneity of intervention effects such as obesity exist. 
We still have limited data to conclusively address whether vitamin D supplementation is effective as either a unique drug or an adjuvant to pharmacotherapy for the treatment of depression. Future trials are needed that not only target depressed patients but also consider baseline levels of vitamin D (40) and how vitamin D dosing and mode of delivery may contribute to its effects on depressive symptoms. We found no evidence of prior dosing studies for vitamin $\mathrm{D}$ supplementation in patients with depression, and it may be time to determine the optimal dose before testing such a dose against placebo in a double-blind trial. Adding vitamin D supplementation to the armamentarium of remedies for depression, although tempting, appears premature based on the evidence that has accumulated on this topic thus far.

\section{Supplementary Material}

Refer to Web version on PubMed Central for supplementary material.

\section{Acknowledgments}

Dr. Shaffer received salary support from the National Institutes of Health [grant number K23-HL112850] and the American Heart Association [grant number 12CRP8870004].

\section{Abbreviations}

25(OH)D 25-hydroxyvitamin D

PRISMA Preferred Reporting Items for Systematic Reviews and Meta-Analyses

SD standard deviation

SMD standardized mean difference

IU international units

RCT randomized controlled trial

MDD major depressive disorder

BDI Beck Depression Inventory

CES-D Center for Epidemiologic Studies Depression scale

GDS Geriatric Depression Scale

HDRS Hamilton Depression Rating Scale

MADRS Montgomery-Ashburg Depression Rating Scale

IM intramuscular

\section{References}

1. Lopez AD, Mathers CD, Ezzati M, Jamison DT, Murray CJ. Global and regional burden of disease and risk factors, 2001: systematic analysis of population health data. Lancet. 2006; 367:1747-57. [PMID: 16731270]. [PubMed: 16731270]

2. Cuijpers P, Beekman AT, Reynolds CF III. Preventing depression: a global priority. JAMA. 2012; 307:1033-4. [PMID: 22416097]. [PubMed: 22416097] 
3. Broadhead WE, Blazer DG, George LK, Tse CK. Depression, disability days, and days lost from work in a prospective epidemiologic survey. JAMA. 1990; 264:2524-8. [PMID: 2146410]. [PubMed: 2146410]

4. Pietrzak RH, Kinley J, Afifi TO, Enns MW, Fawcett J, Sareen J. Subsyndromal depression in the United States: prevalence, course, and risk for incident psychiatric outcomes. Psychol Med. Oct. 2012 31:1-14. [PMID: 23111093].

5. Berk M, Sanders KM, Pasco JA, Jacka FN, Williams LJ, Hayles AL, Dodd S. Vitamin D deficiency may play a role in depression. Med Hypotheses. 2007; 69:1316-9. [PMID: 17499448]. [PubMed: 17499448]

6. Anglin RE, Samaan Z, Walter SD, McDonald SD. Vitamin D deficiency and depression in adults: systematic review and meta-analysis. Br J Psychiatry. 2013; 202:100-7. [PMID: 23377209]. [PubMed: 23377209]

7. Holick MF. Vitamin D deficiency. N Engl J Med. 2007; 357:266-81. [PMID: 17634462]. [PubMed: 17634462]

8. Penckofer S, Kouba J, Byrn M, Estwing Ferrans C. Vitamin D and depression: where is all the sunshine? Issues Ment Health Nurs. 2010; 31:385-93. [PMID: 20450340]. [PubMed: 20450340]

9. Bertone-Johnson ER. Vitamin D and the occurrence of depression: causal association or circumstantial evidence? Nutr Rev. 2009; 67:481-92. [PMID: 19674344]. [PubMed: 19674344]

10. Higgins, JPT.; Green, S., editors. Cochrane Handbook for Systematic Reviews of Interventions Version 5.1.0 [updated March 2011]. The Cochrane Collaboration; 2011. at http://www.cochranehandbook.org [May 29, 2013]

11. Liberati A, Altman DG, Tetzlaff J, Mulrow C, Gotzsche PC, Ioannidis JP, Clarke M, Devereaux PJ, Kleijnen Moher D. The PRISMA statement for reporting systematic reviews and meta-analyses of studies that evaluate health care interventions: explanation and elaboration. Ann Intern Med. 2009; 151:W65-W94. [PMID: 19622512]. [PubMed: 19622512]

12. Khan A, Brodhead AE, Kolts RL, Brown WA. Severity of depressive symptoms and response to antidepressants and placebo in antidepressant trials. J Psychiatr Res. 2005; 39:145-50. [PMID: 15589562]. [PubMed: 15589562]

13. Higgins, JPT.; Altman, DG. Assessing risk of bias in included studies.. In: Higgins, JPT.; Green, S., editors. Cochrane Handbook for Systematic Reviews of Interventions Version 5.1.0 [updated March 2011]. The Cochrane Collaboration; 2011. at http://www.cochrane-handbook.org. [May 29, 2013]

14. Jorde R, Sneve M, Figenschau Y, Svartberg J, Waterloo K. Effects of vitamin D supplementation on symptoms of depression in overweight and obese subjects: randomized double blind trial. $\mathrm{J}$ Intern Med. 2008; 264:599-609. [PMID: 18793245]. [PubMed: 18793245]

15. Hogie-Lorenzen, TL. The Relationship Between Depression Levels and Vitamin D Status Among Older Adults in Eastern South Dakota [master's thesis]. South Dakota State University; Madison, South Dakota: 2003.

16. Yesavage JA, Brink TL, Rose TL, Lum O, Huang V, Adey M, Leirer VO. Development and validation of a geriatric depression screening scale: a preliminary report. J Psychiatr Res. 1982; 17(1):37-49. [PMID: 7183759]. [PubMed: 7183759]

17. Beck AT, Ward CH, Mendelson M, Mock J, Erbaugh J. An inventory for measuring depression. Arch Gen Psychiatry. 1961; 4:561-71. [PMID: 13688369]. [PubMed: 13688369]

18. Mozaffari-Khosravi H, Nabizade L, Yassini-Ardakani SM, Hadinedoushan H, Barzegar K. The effect of 2 different single injections of high dose of vitamin $\mathrm{d}$ on improving the depression in depressed patients with vitamin D deficiency: a randomized clinical trial. J Clin Psychopharmacol. 2013; 33:378-85. [PMID: 23609390]. [PubMed: 23609390]

19. Borenstein, M.; Hedges, L.; Higgins, J.; Rothstein, H. Comprehensive Meta-analysis Version 2. Biostat; Englewood, NJ: 2005.

20. Sievenpiper JL, de Souza RJ, Mirrahimi A, Yu ME, Carleton AJ, Beyene J, Chiavarole L, Di Buono M, Jenkins AL, Leiter LA, Wolever TM, Kendall CW, Jenkins DJ. Effect of fructose on body weight in controlled feeding trials: a systematic review and meta-analysis. Ann Intern Med. 2012; 156:291-304. [PMID: 22351714]. [PubMed: 22351714] 
21. Cohen, J. Statistical Power Analysis for the Behavioral Sciences. 2nd ed.. Lawrence Erlbaum; Hillsdale, NJ: 1988.

22. Beck AT, Steer RA, Carbin MG. Psychometric properties of the Beck Depression Inventory: twenty-five years of evaluation. Clin Psychol Rev. 1988; 8:77-100.

23. Montorio I, Izal M. The Geriatric Depression Scale: a review of its development and utility. Int Psychogeriatr. 1996; 8:103-12. [PMID: 8805091]. [PubMed: 8805091]

24. Macaskill P, Walter SD, Irwig L. A comparison of methods to detect publication bias in metaanalysis. Stat Med. 2001; 20:641-54. [PMID: 11223905]. [PubMed: 11223905]

25. Rosenthal R. The file drawer problem and tolerance for null results. Psychol Bull. 1979; 86:63841.

26. Khoraminya N, Tehrani-Doost M, Jazayeri S, Hosseini A, Djazayery A. Therapeutic effects of vitamin $\mathrm{D}$ as adjunctive therapy to fluoxetine in patients with major depressive disorder. Aust $\mathrm{N} \mathrm{Z}$ J Psychiatry. 2013; 47:271-5. [PMID: 23093054]. [PubMed: 23093054]

27. Bertone-Johnson ER, Powers SI, Spangler L, Larson J, Michael YL, Millen AE, Buesche MN, Salmoirago-Blotcher E, Wassertheil-Smoller S, Brunner RL, Ockene I, Ockene JK, Liu S, Manson JE. Vitamin D supplementation and depression in the Women's Health Initiative Calcium and Vitamin D Trial. Am J Epidemiol. 2012; 176:1-13. [PMID: 22573431]. [PubMed: 22573431]

28. Dean AJ, Bellgrove MA, Hall T, Phan WM, Eyles DW, Kvaskoff D, McGrath JJ. Effects of vitamin D supplementation on cognitive and emotional functioning in young adults--a randomised controlled trial. PLoS ONE. 2011; 6:e25966. [PMID: 22073146]. [PubMed: 22073146]

29. Kjaergaard M, Waterloo K, Wang CE, Almas B, Figenschau Y, Hutchinson MS, Svartberg J, Jorde R. Effect of vitamin D supplement on depression scores in people with low levels of serum 25hydroxyvitamin D: nested case-control study and randomised clinical trial. Br J Psychiatry. 2012; 201:360-8. [PMID: 22790678]. [PubMed: 22790678]

30. Shipowick CD, Moore CB, Corbett C, Bindler R. Vitamin D and depressive symptoms in women during the winter: a pilot study. Appl Nurs Res. 2009; 22:221-5. [PMID: 19616172]. [PubMed: 19616172]

31. Dumville JC, Miles JNV, Porthouse J, Cockayne S, Saxon L, King C. Can vitamin D supplementation prevent winter-time blues? a randomised trial among older women. J Nutr Health Aging. 2006; 10:151-3. [PMID: 16554952]. [PubMed: 16554952]

32. Stokes CS, Baus C, Riemenschneider M, Lammert F. Vitamin D and depression in chronic liver disease. Z Gastroenterol. 2012; 50:3-37.

33. Eyles DW, Smith S, Kinobe R, Hewison M, McGrath JJ. Distribution of the vitamin D receptor and 1 alpha-hydroxylase in human brain. J Chem Neuroanat. 2005; 29:21-30. [PMID: 15589699]. [PubMed: 15589699]

34. Chan R, Chan D, Woo J, Ohlsson C, Mellstrom D, Kwok T, Leung P. Association between serum 25-hydroxyvitamin D and psychological health in older Chinese men in a cohort study. J Affect Disord. 2011; 130:251-9. [PMID: 21056909]. [PubMed: 21056909]

35. May HT, Bair TL, Lappe DL, Anderson JL, Horne BD, Carlquist JF, Muhlestein JB. Association of vitamin D levels with incident depression among a general cardiovascular population. Am Heart J. 2010; 159:1037-43. [PMID: 20569717]. [PubMed: 20569717]

36. Milaneschi Y, Shardell M, Corsi AM, Vazzana R, Bandinelli S, Guralink JM, Ferrucci L. Serum 25-hydroxyvitamin D and depressive symptoms in older women and men. J Clin Endocrinol Metab. 2010; 95:3225-33. [PMID: 20444911]. [PubMed: 20444911]

37. Appleton KM, Rogers PJ, Ness AR. Updated systematic review and meta-analysis of the effects of n-3 long-chain polyunsaturated fatty acids on depressed mood. Am J Clin Nutr. 2010; 91:757-70. [PMID: 20130098]. [PubMed: 20130098]

38. Martins JG. EPA but not DHA appears to be responsible for the efficacy of omega-3 long chain polyunsaturated fatty acid supplementation in depression: evidence from a meta-analysis of randomized controlled trials. J Am Coll Nutr. 2009; 28:525-42. [PMID: 20439549]. [PubMed: 20439549]

39. Bischoff-Ferrari HA, Willett WC, Orav EJ, Lips P, Meunier PJ, Lyons RA, Flicker L, Wark J, Jackson RD, Cauley JA, Meyer HE, Pfeifer M, Sanders KM, Stahelin HB, Theiler R, Dawson- 
Hughes B. A pooled analysis of vitamin D dose requirements for fracture prevention. N Eng J Med. 2012; 367:40-9. [PMID: 22762317].

40. Heaney RP. Vitamin D-baseline status and effective dose. N Engl J Med. 2012; 367:77-8. [PMID: 22762324]. [PubMed: 22762324] 


\author{
Study, Year (Reference) \\ Dean et al., 2011 (28) \\ Bertone-Johnson et al., 2012 (27) \\ Jorde et al., 2008 (14) \\ Hogie-Lorenzen et al., 2003 (15) \\ Kjaergaard et al., 2012 (29) \\ Mozaffari-Khosravi et al., 2013 (18) \\ Khoraminya et al., 2012 (26) \\ Random-effects model for all studies
}

\author{
Nondepressed \\ Dean et al., 2011 (28) \\ Bertone-Johnson et al., 2012 (27) \\ Jorde et al., 2008 (14) \\ Hogie-Lorenzen et al., 2003 (15) \\ Kjaergaard et al., 2012 (29) \\ Random-effects model for subgroup \\ Depressed \\ Mozaffari-Khosravi et al., 2013 (18) \\ Khoraminya et al., 2012 (26)
}

Random-effects model for subgroup

\section{Sufficient Vitamin D}

Dean et al., 2011 (28)

Bertone-Johnson et al., 2012 (27)

Jorde et al., 2008 (14)

Khoraminya et al., 2012 (26)

Random-effects model for subgroup
Insufficient Vitamin D

Hogie-Lorenzen et al., 2003 (15)

Kjaergaard et al., 2012 (29)

Mozaffari-Khosravi et al., 2013(18)

Random-effects model for subgroup
Mean Difference in Depressive Symptoms (95\% CI)

$-0.12(-0.46$ to 0.23$)$

$\mathbf{Z}$

$\mathbf{Z}$

\begin{tabular}{ll}
$-0.66 \quad 0.51$ \\
\hline
\end{tabular}

$0.06(-0.02$ to 0.14$)$

$-0.29(-0.52$ to -0.06$)$

$0.15(-0.28$ to 0.58$)$

$0.01(-0.25$ to 0.27$)$

-0.35 ( -0.76 to 0.06$)$

$-0.96(-1.62$ to -0.31$)$

$\mathbf{- 0 . 1 4}(-0.33$ to 0.05$)$

$-0.12(-0.46$ to 0.23$)$

$0.06(-0.02$ to 0.14$)$

$-0.29(-0.52$ to -0.06$)$

$0.15(-0.28$ to 0.58$)$

0.01 (-0.25 to 0.27$)$

$\mathbf{- 0 . 0 4}(-\mathbf{0 . 2 0}$ to 0.12$)$

$-0.35(-0.76$ to 0.06$)$

$-0.96(-1.62$ to -0.31$)$

$\mathbf{- 0 . 6 0}(-1.19$ to -0.01$)$

$-0.12(-0.46$ to 0.23$)$

$0.06(-0.02$ to 0.14$)$

$-0.29(-0.52$ to -0.06$)$

$-0.96(-1.62$ to -0.31$)$

$\mathbf{- 0 . 2 2}(-\mathbf{0 . 5 3}$ to 0.08$)$

$0.15(-0.28$ to 0.58$)$

$0.01(-0.25$ to 0.27$)$

-0.35 (-0.76 to 0.06$)$

$-0.05(-0.31$ to 0.20$)$ $\begin{array}{ll}1.37 & 0.17\end{array}$

$-2.51 \quad 0.01$

$0.69 \quad 0.49$

$0.09 \quad 0.93$

$-2.89 \quad 0.00$

$-1.41 \quad 0.16$

$-0.66 \quad 0.51$

$1.37 \quad 0.17$

$-2.51 \quad 0.01$

$0.69 \quad 0.49$

$0.09 \quad 0.93$

$-\mathbf{0 . 5 1} 0.61$

$-1.68 \quad 0.09$

$-2.89 \quad 0.00$

$\mathbf{- 1 . 9 9} 0.046$

$-0.66 \quad 0.51$

$1.37 \quad 0.17$

$-2.51 \quad 0.01$

$-2.89 \quad 0.00$

$-1.43 \quad 0.15$

$0.69 \quad 0.49$

$0.09 \quad 0.93$

$-1.68 \quad 0.09$

$-0.40 \quad 0.69$
$-1.68 \quad 0.09$

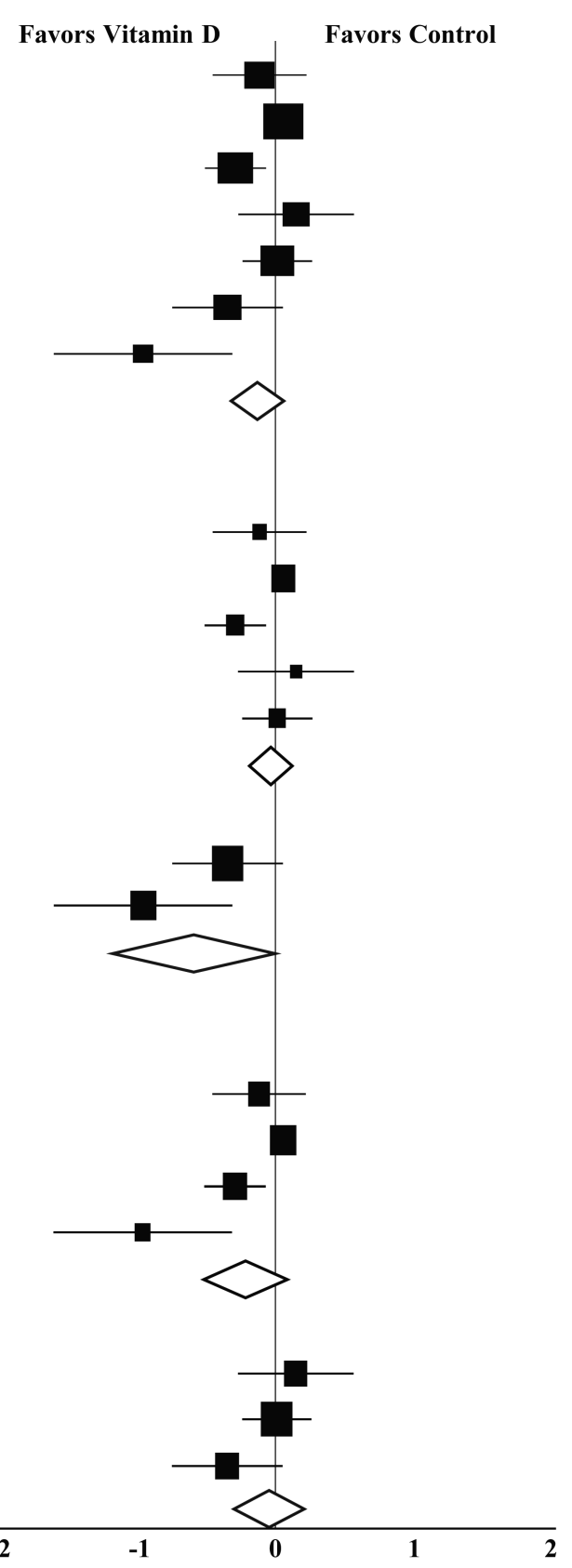

Mean difference (95\% CI) in depressive symptoms

Figure 1.

Forest plots of randomized controlled trials investigating the effect of vitamin D supplementation on depressive symptoms, overall and by depression and vitamin D status. Five weighted pooled intervention effect estimates (diamonds) are shown: one for the full set of 7 trials (overall) and one each for nondepressed participants, depressed participants, participants with sufficient vitamin D, and participants with insufficient vitamin D. Data are expressed as standardized mean differences with $95 \%$ CI, using the inverse-variance method and random-effects models. Trials categorized as "nondepressed" did not specifically recruit participants with depression or included participants whose baseline depressive symptom scores were indicative of no or mild depression; trials categorized as "depressed" included participants with clinically significant depressive symptoms and/or major 
depressive disorder. Categorization of trials as "sufficient vitamin D" or "insufficient vitamin D" was based on participants' baseline concentrations of 25-hydroxyvitamin D and established cutpoints for interpreting these concentrations (7). 


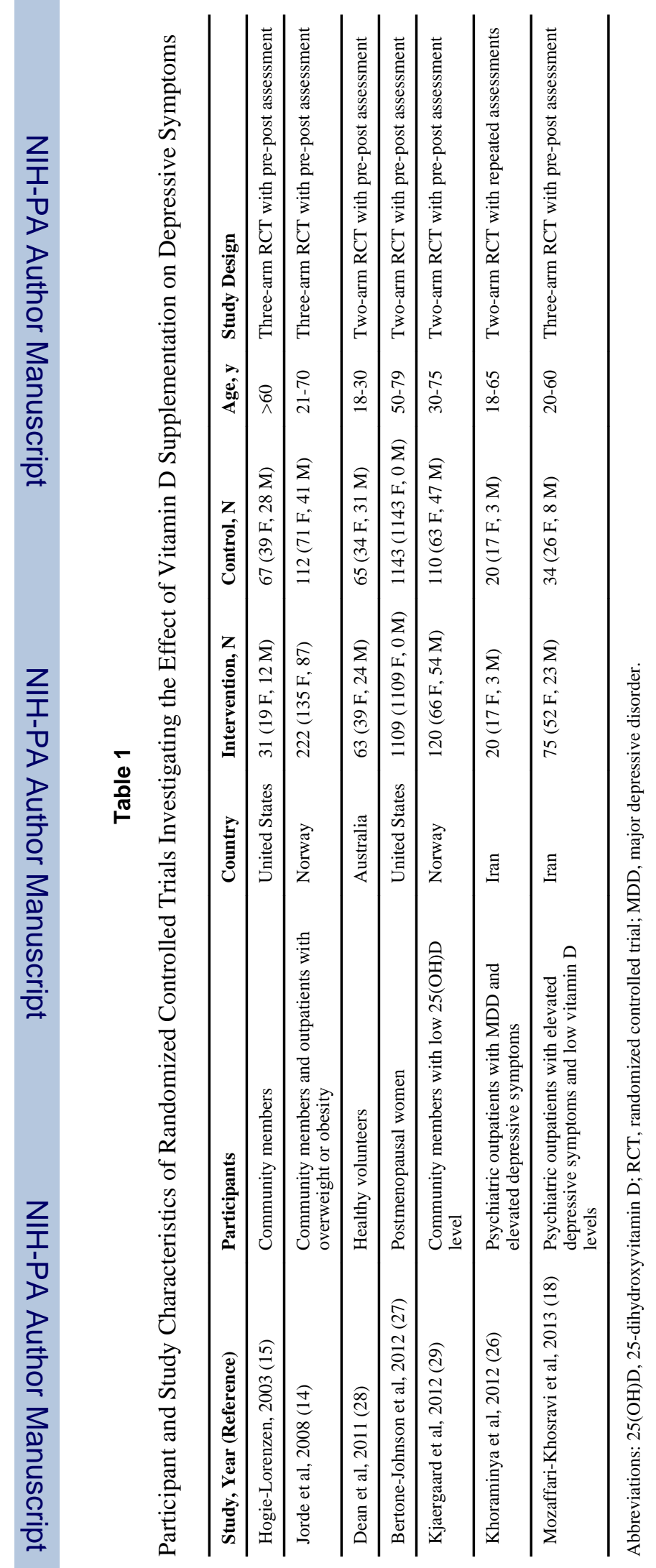




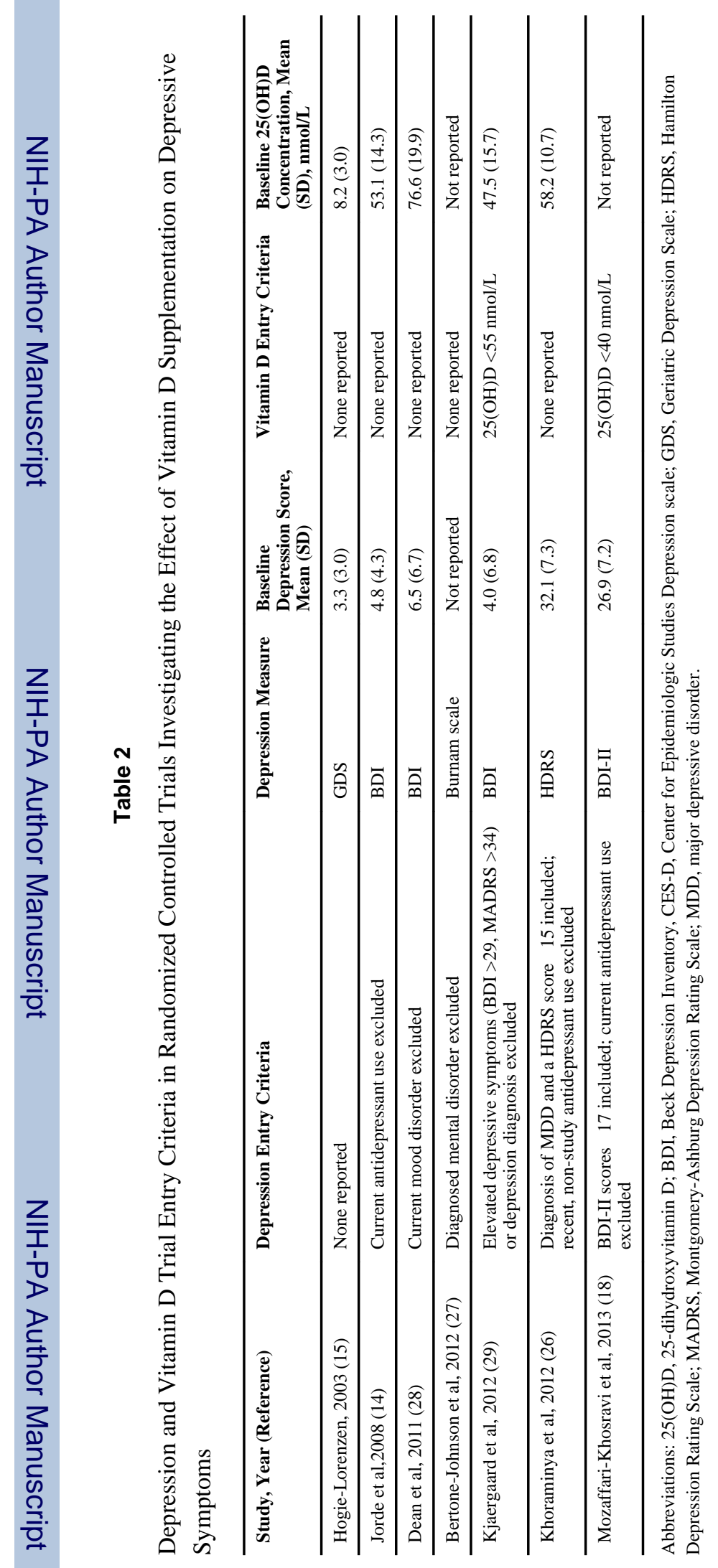




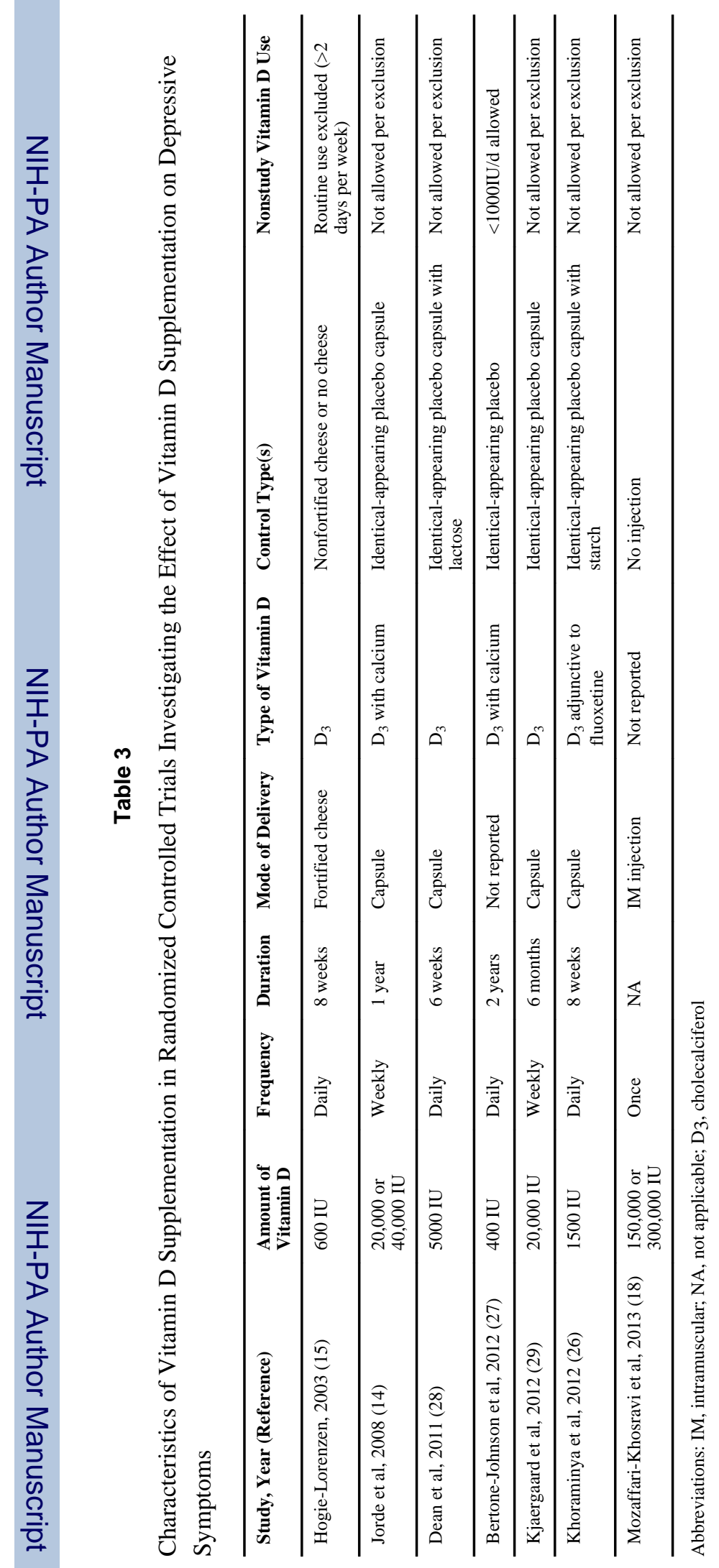

